\title{
Usefulness of Stress Myocardial Perfusion Imaging and Baseline Clinical Factors for Predicting Cardiovascular Events in Patients With Prior Coronary Artery Disease
}

\author{
Tatsuhiko Furuhashi, MD; Masao Moroi, MD; Toru Awaya, MD; Megumi Minakawa, MD; \\ Hirofumi Masai, MD; Taeko Kunimasa, MD; Hiroshi Fukuda, MD
}

\begin{abstract}
Background: Stress myocardial perfusion imaging (MPI) is a well-established diagnostic and prognostic tool for coronary artery disease (CAD). However, fixed and/or reversible stress MPI defects vary considerably among patients with CAD. The usefulness of stress MPI and baseline clinical factors as independent predictors of cardiovascular events in patients with prior CAD were assessed.
\end{abstract}

\begin{abstract}
Methods and Results: Stress MPI was performed in 354 patients with prior CAD. Their mean age was 71 years; the mean duration from first revascularization and/or myocardial infarction (MI) onset until stress MPI was 7.1 years; and the mean follow-up period was 34 months. Cardiovascular events were observed in 100 patients (28\%), and were categorized as cardiac-related death, non-fatal $\mathrm{MI}$, unstable angina, heart failure and late ( $>2$ months from stress MPI) coronary revascularization. Multivariate Cox regression analysis revealed that peripheral arterial disease (hazard ratio $[H R]=2.95 ; P<0.001)$, current smoking $(H R=2.36 ; P=0.006)$, chronic kidney disease $(H R=2.15$; $P<0.001)$, left ventricular ejection fraction ( $H R=0.98 ; P=0.017)$, and grade of myocardial ischemia assessed by stress MPI $(H R=1.14 ; P=0.007)$ were independent and significant predictors of cardiovascular events.
\end{abstract}

Conclusions: In patients with prior CAD, baseline clinical factors and grade of myocardial ischemia assessed by stress MPI results are significant predictors of cardiovascular events. (Circ $J$ 2014; 78: 1676-1683)

Key Words: Cardiovascular events; Myocardial ischemia; Prior coronary artery disease; Stress myocardial perfusion imaging

$\mathbf{T}$ he usefulness of stress myocardial perfusion imaging (MPI) for the diagnosis of coronary artery disease (CAD) and assessment of cardiovascular prognosis is well established. ${ }^{1-13}$ In general, normal stress MPI results are correlated with excellent cardiovascular prognosis, as evidenced by the annual cardiovascular event rate of $<1 \% .{ }^{14}$ However, fixed and/or reversible stress MPI defects vary considerably among patients with prior CAD. Although several prognostic studies have been reported, the predictive value of stress MPI results for cardiovascular prognosis still remains unclear in patients with prior CAD..$^{15-17}$ The present study assessed the usefulness of stress MPI results and baseline clinical factors as independent predictors of cardiovascular prognosis in patients with prior CAD.

\section{Methods}

\section{Patients and the Study Protocol}

We designed a retrospective study of patients who had under- gone stress MPI. In total, 1,014 consecutive patients with suspected or prior CAD had undergone thallium-201stress MPI between 2008 and 2010. The following patients were excluded: 154 with no prognostic data following stress MPI, 490 with no prior $\mathrm{CAD}, 10$ with significant ischemia [summed difference score (SDS) of $\geq 2$ on stress MPI] who achieved revascularization within 2 months of subsequent percutaneous coronary intervention, ${ }^{4} 4$ with severe valvular disease requiring surgery, and 2 with aortic disease requiring surgery. Finally, 354 patients with prior CAD were included in the study. No patient had experienced new-onset $\mathrm{CAD}$ or had undergone invasive therapy for CAD within 12 months before undergoing stress MPI.

The study protocol was approved by the Committee on Human Investigation of the Toho University Ohashi Medical Center (approval No.12-62), and the study was conducted in accordance with the ethical standards of the 1964 Declaration of Helsinki (as revised in Tokyo in 2004) and subsequent revisions. All included patients provided informed consent.

Information about prior CAD was obtained from clinical

Received January 6, 2014; revised manuscript received March 31, 2014; accepted April 9, 2014; released online May 21, 2014 Time for primary review: 32 days

Division of Cardiovascular Medicine, Toho University Ohashi Medical Center, Tokyo, Japan

Mailing address: Masao Moroi, MD, FACP, Division of Cardiovascular Medicine, Toho University Ohashi Medical Center, 2-17-6 Ohashi, Meguro-ku, Tokyo 153-8515, Japan. E-mail: moroi@med.toho-u.ac.jp

ISSN-1346-9843 doi:10.1253/circj.CJ-14-0018

All rights are reserved to the Japanese Circulation Society. For permissions, please e-mail: cj@j-circ.or.jp 


\begin{tabular}{|c|c|c|c|c|}
\hline & Overall $(n=354)$ & Events $(+)(n=100)$ & Events $(-)(n=254)$ & $P$ value \\
\hline Age & $71 \pm 10$ & $74 \pm 9.4$ & $70 \pm 11$ & 0.009 \\
\hline Male & 281 (79\%) & $83(83 \%)$ & $198(78 \%)$ & 0.31 \\
\hline Hypertension & $311(88 \%)$ & $92(92 \%)$ & $219(86 \%)$ & 0.15 \\
\hline Diabetes & $125(35 \%)$ & $46(46 \%)$ & $79(31 \%)$ & 0.010 \\
\hline Hyperlipidemia & 279 (79\%) & $75(75 \%)$ & 204 (80\%) & 0.31 \\
\hline CKD & $126(36 \%)$ & $54(54 \%)$ & $72(28 \%)$ & $<0.001$ \\
\hline History of smoking & $215(61 \%)$ & 59 (59\%) & $156(61 \%)$ & 0.72 \\
\hline Current smoking & $31(9 \%)$ & $16(16 \%)$ & $15(6 \%)$ & 0.006 \\
\hline Familial history of CAD & $64(18 \%)$ & $21(21 \%)$ & $43(17 \%)$ & 0.36 \\
\hline CVD & $44(12 \%)$ & $17(17 \%)$ & $27(11 \%)$ & 0.11 \\
\hline PAD & $47(13 \%)$ & $32(32 \%)$ & $15(6 \%)$ & $<0.001$ \\
\hline Duration from first revascularization or onset of MI (years) & $7.1 \pm 5.9$ & $7.4 \pm 5.7$ & $7.0 \pm 5.9$ & 0.57 \\
\hline Previous MI & $179(51 \%)$ & $53(53 \%)$ & $126(50 \%)$ & 0.64 \\
\hline Coronary angioplasty & $335(95 \%)$ & $96(96 \%)$ & $239(94 \%)$ & 0.61 \\
\hline $\mathrm{PCl}$ & $312(88 \%)$ & $92(92 \%)$ & $220(87 \%)$ & 0.44 \\
\hline CABG & $46(13 \%)$ & $11(11 \%)$ & $35(14 \%)$ & 0.38 \\
\hline \multicolumn{5}{|l|}{ Laboratory data } \\
\hline Haemoglobin & $12.9 \pm 1.9$ & $12.2 \pm 2.0$ & $13.2 \pm 1.8$ & $<0.001$ \\
\hline Albumin & $4.1 \pm 0.4$ & $4.1 \pm 0.5$ & $4.2 \pm 0.4$ & 0.11 \\
\hline LDL cholesterol & $93 \pm 27$ & $97 \pm 28$ & $92 \pm 26$ & 0.19 \\
\hline HDL cholesterol & $53 \pm 14$ & $51 \pm 14$ & $54 \pm 14$ & 0.15 \\
\hline LDL/HDL & $1.9 \pm 0.7$ & $2.0 \pm 0.8$ & $1.8 \pm 0.6$ & 0.09 \\
\hline BNP & $118 \pm 231$ & $231 \pm 356$ & $66 \pm 112$ & 0.014 \\
\hline \multicolumn{5}{|l|}{ Echocardiography } \\
\hline LVEF & $61 \pm 12$ & $57 \pm 15$ & $62 \pm 11$ & 0.015 \\
\hline Adenosine stress test & 201 (57\%) & $70(70 \%)$ & $131(52 \%)$ & 0.002 \\
\hline \multicolumn{5}{|l|}{ Stress MPI } \\
\hline SSS & $4.2 \pm 5.1$ & $4.8 \pm 4.9$ & $4.0 \pm 5.2$ & 0.013 \\
\hline SRS & $3.4 \pm 5.0$ & $3.4 \pm 4.6$ & $3.4 \pm 5.2$ & 0.46 \\
\hline SDS & $0.8 \pm 1.7$ & $1.4 \pm 2.3$ & $0.5 \pm 1.3$ & 0.001 \\
\hline \multicolumn{5}{|l|}{ Medication } \\
\hline Aspirin & 309 (87\%) & $88(88 \%)$ & $221(87 \%)$ & 0.86 \\
\hline Clopidogrel & $90(25 \%)$ & $19(19 \%)$ & $71(28 \%)$ & 0.10 \\
\hline ACE-I/ARB & 285 (81\%) & $79(79 \%)$ & 206 (81\%) & 0.65 \\
\hline$\beta$-blockers & $108(31 \%)$ & $34(34 \%)$ & $74(29 \%)$ & 0.44 \\
\hline $\mathrm{CCB}$ & $150(42 \%)$ & $51(51 \%)$ & 99 (39\%) & 0.06 \\
\hline Nitrates & $72(20 \%)$ & $27(27 \%)$ & $45(18 \%)$ & 0.06 \\
\hline Nicorandil & $40(11 \%)$ & $15(15 \%)$ & $25(10 \%)$ & 0.19 \\
\hline Diuretics & 77 (22\%) & $35(35 \%)$ & $42(17 \%)$ & $<0.001$ \\
\hline Statin & $253(71 \%)$ & $62(62 \%)$ & $191(75 \%)$ & 0.018 \\
\hline
\end{tabular}

ACE-I, angiotensin-converting enzyme inhibitors; ARB, aldosterone receptor blockers; BNP, brain natriuretic peptide; CABG, coronary artery bypass grafting; CAD, coronary artery disease; CCB, calcium channel blockers; CKD, chronic kidney disease; CVD, cerebral vascular disease; HDL, high-density lipoprotein; LDL, low-density lipoprotein; LVEF, left ventricular ejection fraction; MI, myocardial infarction; MPI, myocardial perfusion imaging; PAD, peripheral artery disease; PCI, percutaneous coronary intervention; SDS, summed difference score; SRS, summed rest score; SSS, summed stress score.

records or patient interviews. Of the 354 patients, $335(95 \%)$ experienced coronary revascularization and $19(5 \%)$ experienced myocardial infarction (MI) without coronary revascularization. We obtained information regarding the duration from coronary revascularization or onset of MI to stress MPI. In addition, data on age, gender, medication usage, and other coronary risk factors were routinely collected during stress MPI. The following were considered possible cardiovascular risk factors: cigarette smoking (current and past), history of hypertension, diabetes, hyperlipidemia, chronic kidney disease (CKD), and prior $\mathrm{CAD}$ in a first-degree relative (aged $<55$ years in males and aged $<65$ years in females). Hypertension was defined as a systolic blood pressure of $>140 \mathrm{mmHg}$, diastolic blood pressure of $>90 \mathrm{mmHg}$, and/or current treatment with antihypertensives. Diabetes was defined as a fasting blood glucose level of $>126 \mathrm{mg} / \mathrm{dl}$, a glycosylated hemoglobin level of $>6.5 \%$ based on the National Glycohemoglobin Standardization Program definition, or current treatment with insulin or oral antidiabetic medications. Hyperlipidemia was defined as the presence of hypercholesterolemia (total cholesterol level of $>220 \mathrm{mg} / \mathrm{dl}$ ), hypertriglyceridemia (serum triglyceride level of $>150 \mathrm{mg} / \mathrm{dl}$ ), and/or current lipid-lowering therapy. CKD was 
defined according to the National Kidney Foundation criteria ${ }^{18}$ as an estimated glomerular filtration rate of $<60 \mathrm{ml} \cdot \mathrm{min}^{-1} \cdot 1.73 \mathrm{~m}^{-2}$ and/or persistent proteinuria for at least 3 months. The left ventricular ejection fraction (LVEF) was measured by motion (M)-mode echocardiography within 1 month of stress MPI. Peripheral arterial disease (PAD) was defined as a previous medical history of angioplasty for peripheral arteries and/or an ankle brachial index (ABI) of $<0.9 .1^{19,20}$

\section{Stress MPI}

Exercise or pharmacological stress tests were performed. Patients were suspended from cardiac medication usage and caffeine ingestion for 1 day before examination. All exercise tests were performed using a treadmill. No patient underwent ergometer exercise stress testing. The treadmill exercise test (Bruce protocol) was considered adequate if patients achieved $85 \%$ of the maximum predicted heart rate and/or developed chest pain. Patients with limited exercise capacity (who failed to achieve $85 \%$ of the predicted heart rate during the treadmill test) underwent a pharmacological stress test that consisted of intravenous adenosine infusion. Adenosine and thallium-201 were administered in separate arms. An automated infusion pump was used to deliver intravenous adenosine infusion $\left(0.120 \mathrm{mg} \cdot \mathrm{kg}^{-1} \cdot \mathrm{min}^{-1}\right)$ over $6 \mathrm{~min}$. Thallium-201 (111 MBq; Fujifilm RI Pharma Co, Ltd, Tokyo, Japan) was injected into a peripheral vein either 1 min before cessation of the treadmill stress test or $3 \mathrm{~min}$ after initiation of adenosine infusion.

All myocardial perfusion single-photon emission computed tomography (SPECT) data were acquired using a 3-headed gamma camera (MS-3; Siemens, Chicago, IL, USA) equipped with a low-energy cardiofocal collimator and a computer interface (ICON; Siemens). Stress SPECT was performed $10 \mathrm{~min}$ after stress testing and rest SPECT was performed $4 \mathrm{~h}$ after stress imaging. In total, 90 projections were obtained for $20 \mathrm{~s}$ each in $4 / 360^{\circ}$ intervals and stored on $64 \times 64$ matrices. A $15 \%$ symmetrical energy window centered on the $70-\mathrm{keV}$ peak was used. Tomographic reconstruction was performed by the standard filtered back-projection technique using a Butterworth filter with a cut-off frequency of 0.5 cycles/pixel and an order of 5 . No correction was made for attenuation or scatter.

The SPECT images were reoriented along the short, horizontal, and vertical long axes for analysis. SPECT data analysis was performed on the basis of agreement of among $\geq 2$ experienced nuclear medicine physicians (T.F. and M.M.). Defects were classified as reversible (including partially reversible) or fixed (irreversible). The SPECT images were assessed to determine the presence, location, and severity of any perfusion defect. Observers assessed SPECT images visually and semiquantitatively, and software applications were not used. The left ventricle (LV) was divided into 17 segments and each segment was assigned a score using a 5-point scoring system $(0=$ normal; $1=$ mildly reduced; $2=$ moderately reduced; $3=$ severely reduced; and $4=$ uptake absent). The following scores were calculated: the summed score at stress [summed stress score (SSS)], which showed myocardial ischemia and MI; the summed score at rest [summed rest score (SRS)], which showed MI or prolonged myocardial ischemia; ${ }^{21}$ and the difference between the stress and rest scores (SDS), which showed myocardial ischemia.

\section{End-Points and Follow up}

Follow up commenced after assessment of clinical information and stress MPI. Cardiovascular events considered as endpoints were: cardiac death, non-fatal MI, Braunwald class III unstable angina requiring hospitalization, congestive heart fail- ure requiring hospitalization and late ( $>2$ months after stress MPI) coronary revascularization. Cardiac deaths included: sudden cardiac death, fatal MI, death due to heart failure, or death due to arrhythmia. Sudden cardiac death was defined as witnessed cardiac arrest, death within $1 \mathrm{~h}$ of onset of acute symptoms, or unexpected death for those who had been considered well for the previous $24 \mathrm{~h}$. Braunwald class III unstable angina was defined as acute angina at rest within $48 \mathrm{~h}$ of onset. The diagnosis of congestive heart failure was made by the presence of the following findings and by the need for hospitalization and aggressive medical treatment: findings from a careful history taking, typical symptoms (dyspnea or orthopnea), neck vein distention, peripheral edema, lung rales, S3 gallop, and tachycardia, together with chest X-ray findings (cardiomegaly, bilateral lung congestion, and/or pleural effusion). The final diagnosis of congestive heart failure was confirmed after admission using electrocardiography, 2-dimensional echocardiography, and/or chest computed tomography to exclude non-cardiac diseases with similar symptoms or signs. Patients were regularly followed up for a mean duration of $34 \pm 17$ months (range 1-57 months).

\section{Statistical Analysis}

Continuous variables are presented as mean \pm standard deviation and categorical variables as observed number of patients (percentage). To compare patient characteristics between groups, the Mann-Whitney U-test was used for continuous variables and Fisher's exact test was used for categorical variables. Cox regression hazard analysis was used to assess the possible risk factors. Kaplan-Meier event-free curves were generated, and the risks of end-points were compared between patient groups using log-rank tests. Statistical analyses were performed using SPSS statistical software (SPSS Incorporated, Chicago, IL, USA). A $\mathrm{P}$ value of $<0.05$ was considered statistically significant.

\section{Results}

\section{Patient Characteristics}

Table 1 summarizes patient characteristics, comparing the results of patients who developed cardiovascular events with those of patients who did not. Age and frequency of CKD, current smoking, and PAD were significantly higher in patients who developed cardiovascular events. Furthermore, with respect to the laboratory data, hemoglobin levels were significantly lower and plasma brain natriuretic peptide levels were significantly higher in patients with cardiovascular events. On echocardiography, LVEF was relatively preserved overall; however, patients who developed cardiovascular events had lower LVEFs. The frequency of adenosine stress testing was significantly higher in patients who developed cardiovascular events. Regarding stress MPI results, SSS and SDS were significantly higher in those who developed cardiovascular events. With respect to medication use, diuretic use was significantly higher and statin use was significantly lower in patients who developed cardiovascular events. No significant difference was observed in the mean duration from first revascularization and/ or MI onset until stress MPI. Among patients with previous MI, the mean SRS was 5.9 \pm 5.8 and the mean LVEF was $57 \pm 13 \%$. The mean duration from onset of MI to stress MPI was $8.4 \pm 6.4$ years, which was significantly longer than the duration of CAD among patients with no previous MI (5.9 \pm 5.0 years, $\mathrm{P}<0.001$ ).

\section{Outcomes}

Cardiovascular events occurred in 100 patients with prior 


\begin{tabular}{|lccc|}
\hline Table 2. Univariate Cox Regression Analysis & & & \\
Male & Hazard ratio & $95 \%$ Cl & P value \\
Age & 1.26 & $0.75-2.13$ & 0.38 \\
Duration from first revascularization or onset of MI (years) & 1.04 & $1.02-1.06$ & $<0.001$ \\
Previous MI & 1.01 & $0.98-1.04$ & 0.61 \\
CVD & 1.06 & $0.72-1.57$ & 0.76 \\
PAD & 1.53 & $0.91-2.58$ & 0.11 \\
Hypertension & 4.78 & $3.12-7.30$ & $<0.001$ \\
Diabetes & 1.85 & $0.90-3.82$ & 0.09 \\
CKD & 1.70 & $1.15-2.52$ & 0.008 \\
Current smoking & 2.62 & $1.77-3.89$ & $<0.001$ \\
History of smoking & 2.34 & $1.37-4.00$ & 0.002 \\
Laboratory data & 1.13 & $0.76-1.69$ & 0.54 \\
Hemoglobin & & & \\
LDL/HDL & 0.79 & $0.71-0.87$ & $<0.001$ \\
BNP & 1.55 & $1.17-2.07$ & 0.003 \\
LVEF on echocardiography & 1.00 & $1.00-1.00$ & 0.05 \\
Adenosine stress test & 0.97 & $0.96-0.99$ & $<0.001$ \\
Stress MPI & 2.15 & $1.40-3.30$ & $<0.001$ \\
SSS & & & \\
SRS & 1.02 & $0.99-1.06$ & 0.19 \\
SDS & 0.99 & $0.95-1.03$ & 0.72 \\
No statin use & 1.29 & $1.19-1.41$ & $<0.001$ \\
\hline Cl confing & 1.95 & $1.30-2.92$ & 0.001 \\
\hline
\end{tabular}

$\mathrm{Cl}$, confidence interval. Other abbreviations as in Table 1.

\begin{tabular}{|lccc|}
\hline Table 3. Multivariate Cox Regression Analysis & & & \\
& Hazard ratio & $\mathbf{9 5 \%} \mathbf{~ C l}$ & P value \\
Age & 1.03 & $1.00-1.05$ & 0.043 \\
PAD & 2.95 & $1.81-4.81$ & $<0.001$ \\
Diabetes & 1.42 & $0.93-2.19$ & 0.11 \\
CKD & 2.15 & $1.39-3.33$ & $<0.001$ \\
Current smoking & 2.36 & $1.28-4.35$ & 0.006 \\
LVEF on echocardiography & 0.98 & $0.97-0.99$ & 0.017 \\
Adenosine stress test & 0.79 & $0.46-1.37$ & 0.40 \\
SDS of stress MPI & 1.14 & $1.04-1.26$ & 0.007 \\
No statin use & 1.53 & $0.98-2.40$ & 0.07 \\
\hline
\end{tabular}

Abbreviations as in Tables 1,2.

CAD (cardiac death in 23 patients, non-fatal MI in 17 patients, Braunwald III unstable angina in 6 patients, congestive heart failure in 30 patients, and remote revascularization in 24 patients). Univariate Cox regression hazard analysis (Table 2) revealed that age, PAD, diabetes, $\mathrm{CKD}$, current smoking, hemoglobin levels, low-density lipoprotein (LDL)/high-density lipoprotein (HDL) ratio, LVEF on echocardiography, adenosine stress testing, SDS on stress MPI and no statin use were significant predictors of cardiovascular events. As previously reported, the minimum number of variables that should be entered into a multivariable model is a function of the number of events that occur during the study. In general, 1 variable for every 10 events is strongly recommended.22 From the Cox univariate regression analysis, we analyzed the relative risk level by multivariate Cox regression hazard analysis for cardiovascular events associated with age, PAD, diabetes, CKD, current smoking, LVEF on echocardiography, adenosine stress test, SDS on stress MPI and no statin use. Age (hazard ratio $[\mathrm{HR}]=1.03 ; \mathrm{P}=0.043), \mathrm{PAD} \quad(\mathrm{HR}=2.95 ; \mathrm{P}<0.001), \mathrm{CKD}$
$(\mathrm{HR}=2.15 ; \mathrm{P}<0.001)$, current smoking $(\mathrm{HR}=2.36 ; \mathrm{P}=0.006)$, LVEF on echocardiography $(\mathrm{HR}=0.98 ; \mathrm{P}=0.017)$ and $\mathrm{SDS}$ on stress MPI $(\mathrm{HR}=1.14 ; \mathrm{P}=0.007)$ were independent and significant risk factors for cardiovascular events (Table 3 ). We then determined the number of coexisting risk factors (PAD, current smoking, and CKD) identified by multivariate Cox regression analysis as follows: no factor: $\mathrm{n}=193,32$ events $(17 \%)$; 1 factor: $n=121,38$ events $(31 \%) ; 2$ factors: $n=39,29$ events (74\%); and 3 factors: $n=1,1$ event $(100 \%)$. The number of coexisting risk factors was identified as a predictor of cardiovascular events $(\mathrm{HR}=2.91 ; \mathrm{P}<0.001)$.

In stress MPI, only SDS was a predictor of cardiovascular events. We defined a SDS of $\geq 2$ as a reversible defect abnormality, on the basis of a prognostic study ${ }^{23}$ and a past study of our institution. ${ }^{24}$ The Kaplan-Meier survival curves shown in Figure 1 revealed superior prognosis (concerning cardiovascular events) in patients with a SDS of $<2$ compared with patients with a SDS of $\geq 2$. The predictive value of the number of coexisting risk factors was confirmed in patients with a SDS 


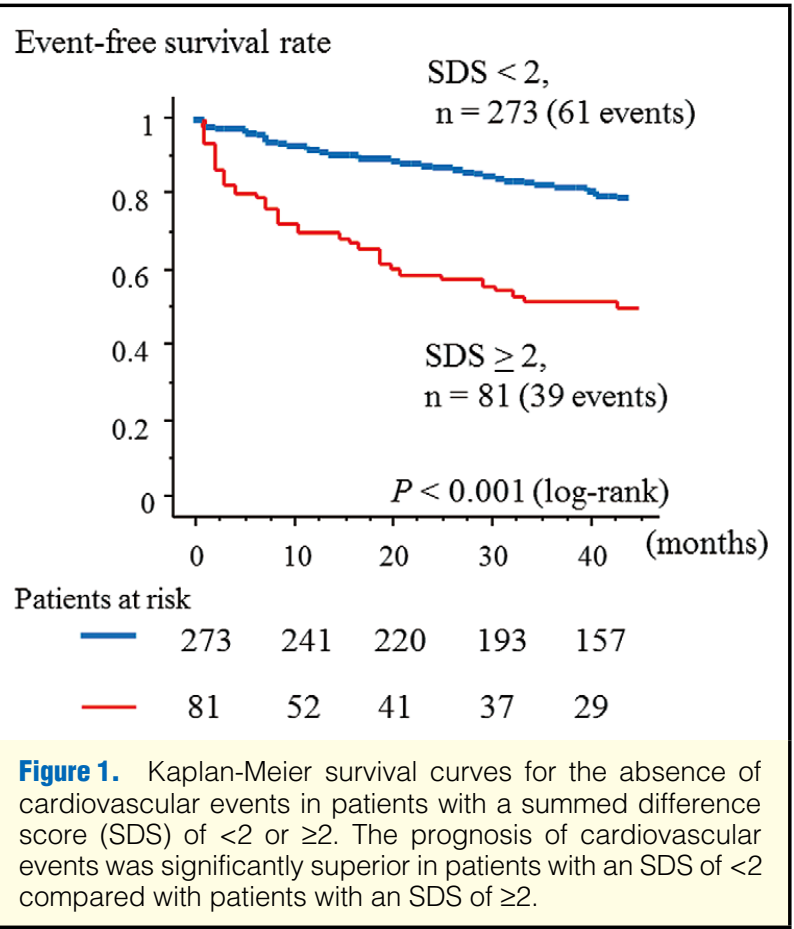

of $<2$ [ $n=273$, no factor: $n=160,22$ events $(14 \%) ; 1$ factor: $n=89$, 22 events $(25 \%)$; 2 factors: $n=23,16$ events $(70 \%) ; 3$ factors: $\mathrm{n}=1,1$ event $(100 \%) ; \mathrm{HR}=3.11 ; \mathrm{P}<0.001]$. The predictive value of the number of coexisting risk factors was also confirmed in patients with a SDS of $\geq 2$ [ $n=81$, no factor: $n=33,10$ events (30\%); 1 factor: $n=32,16$ events $(50 \%) ; 2$ factors: $n=16,13$ events $(81 \%) ; \mathrm{HR}=1.97 ; \mathrm{P}=0.002]$. Figure 2 showed a comparison of the Kaplan-Meier survival curves for patients with different numbers of risk factors ( 0,1 and 2-3 factors). In patients with a SDS of $<2$ and with a SDS of $\geq 2$, the prognosis of cardiovascular events was significantly different, with different numbers of coexisting risk factors.

\section{Discussion}

This study demonstrated that age, PAD, current smoking, CKD, LVEF and grade of reversible defect abnormality assessed by stress MPI results were significant and independent predictors of cardiovascular events in patients with prior CAD. Previous studies have shown that the annual rates of cardiac events are significantly lower in Japan than in the USA. ${ }^{2,5}$ In this study, the number of hard cardiovascular events (cardiac death, nonfatal MI and unstable angina) was low $(n=46)$. In contrast, 30 cases of heart failure and 24 cases of late coronary revascularization were observed. We included heart failure and late coronary revascularization for detailed assessment of cardiac risk factors. The viewpoint of including heart failure and coronary revascularization as end-points of cardiovascular events matches the current Japanese clinical practice, because patients with both heart failure and $\mathrm{CAD}$ require intensive medical care and have a potentially poor prognosis.

Previous studies have reported that PAD is a risk factor for cardiovascular disease. ${ }^{19,20}$ In addition, although a low ABI and a history of angioplasty in the peripheral arteries are both potential predictors of cardiovascular events, some epidemiological studies have shown that patients with a high ABI (related to increased peripheral arterial stiffness) experience in- creased cardiovascular risk. Specifically, the risk of patients with an $\mathrm{ABI}$ of $>1.4$ is considered equivalent to that of patients with an $\mathrm{ABI}$ of $<0.9 .^{20,25}$ In the present study, PAD was simply defined as an ABI of $<0.9$ and/or a history of angioplasty to peripheral arteries. Thus, it is possible that PAD was missed in some patients who had increased or normal ABIs on account of increased arterial stiffness.

Smoking cessation after the onset of CAD is known to decrease the risk of mortality, hospitalization, and $\mathrm{CAD}$ recurrence. ${ }^{26-28}$ Continuous smoking increases the risk of cardiovascular events, particularly in patients with prior CAD. The relationship between CKD and adverse cardiovascular prognosis has been established by large community-based studies. ${ }^{29-32}$

LVEF has been widely used to assess the prognosis of cardiovascular disease, ${ }^{33-36}$ and the values of LVEF measured by echocardiography, electrocardiogram-gated SPECT and left ventriculography are closely correlated. ${ }^{37}$ The present study showed that LVEF assessed by echocardiography could be a predictor of cardiovascular events.

In agreement with our earlier research demonstrating that the number of coexisting cardiac risk factors (PAD, diabetes, and $\mathrm{CKD}$ ) correlates with poor cardiovascular prognosis, even in patients with normal stress MPI results and no prior CAD, ${ }^{38}$ the number of coexisting risk factors itself was a considerable cardiac risk factor in patients with and without reversible defect abnormalities.

Although stress MPI has been established as a strong predictor of cardiovascular events in general, we found that only SDS (grade of myocardial ischemia) was a predictor. Neither SSS (grade of myocardial ischemia and MI) nor SRS (grade of MI or prolonged myocardial ischemia) was a cardiovascular predictor. As shown by previous studies, ${ }^{39-41}$ the cardiovascular event rate might be relatively low in patients without significant reversible defect abnormalities, regardless of MI severity. In this study, no large perfusion defects (mean SSS: 4.2 \pm 5.1 ; SRS: $3.4 \pm 5.0$; and SDS: $0.8 \pm 1.7$ ) were observed; however, this situation might not be close to the real generalized situation for CAD patients. We emphasize our study results demonstrating that the SDS of stress MPI reflects the extent and severity of hemodynamically significant coronary stenosis and the amount of jeopardized myocardium. This is an important factor for assessing cardiovascular prognosis in patients with prior CAD. In a previous prognostic study based on the Japanese Assessment of Cardiac Events and Survival Study by Quantitative Gated SPECT (J-ACCESS) database, assessment of perfusion and function using stress/rest gated myocardial perfusion SPECT, in combination with clinical risk factors, showed independent as well as incremental prognostic value for the prediction of major cardiac events in Japanese patients. Age, diabetes, SSS, end systolic volume, and LVEF were independent and significant predictors of major cardiac events. ${ }^{5}$ In addition, a sub-study based on the J-ACCESS database in patients who had undergone coronary angiography within 3 months before or after stress MPI showed that end systolic volume and SDS were independent and significant predictors of cardiac events. ${ }^{41}$ In a previous follow-up study of 1,988 Japanese patients, although both SSS and SDS were identified as independent predictors of cardiac death, SDS and LVEF were shown to be independent predictors of MI and unstable angina. ${ }^{40}$ Unlike these previous analyses, patients did not show large perfusion defect in stress MPI in the present study. This is the major difference between the findings of the present study and those of past studies. Similar to the present study, the sub-study based on the J-ACCESS database in pa- 


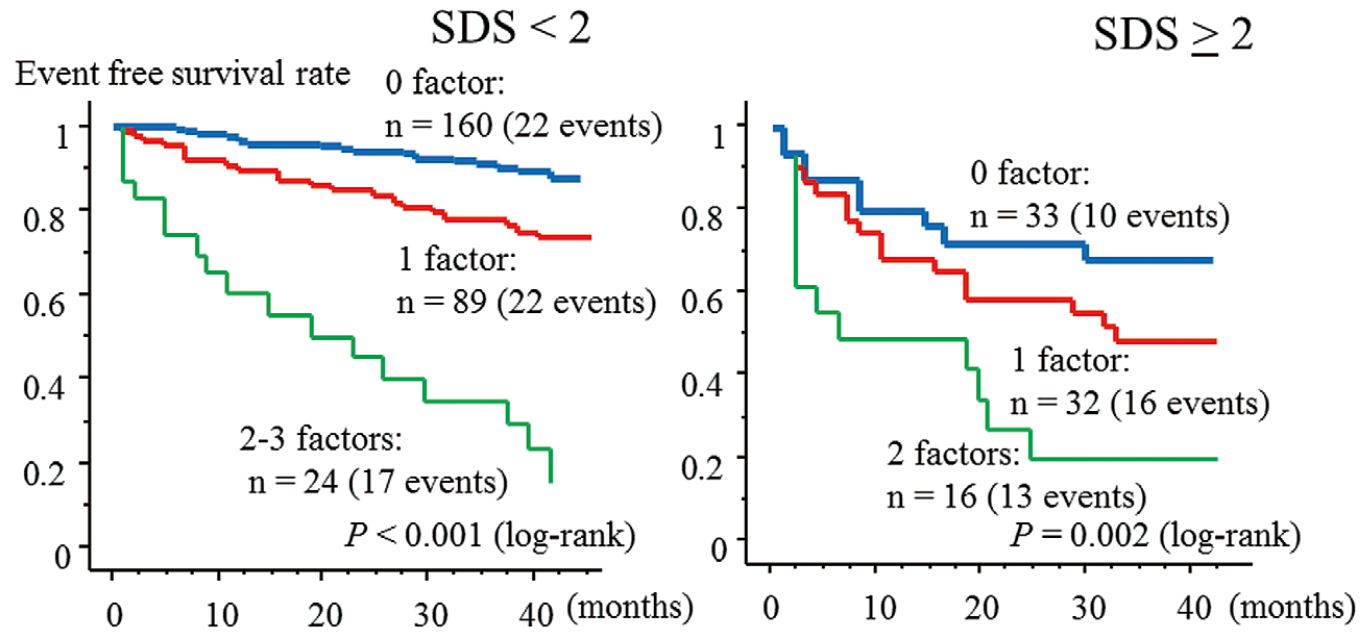

Patients at risk.

$\begin{array}{rrrrrrrrrr}160 & 150 & 140 & 127 & 103 & -33 & 22 & 18 & 17 & 14 \\ -89 & 77 & 70 & 59 & 51 & -32 & 22 & 19 & 17 & 12 \\ -24 & 14 & 10 & 7 & 3 & -16 & 8 & 4 & 3 & 3\end{array}$

Figure 2. Kaplan-Meier survival curves for the absence of cardiovascular events in patients with coexisting clinical risk factors (peripheral arterial disease, current smoking, and chronic kidney disease). In patients with a summed difference score (SDS) of $<2$ and with an SDS of $\geq 2$, the prognosis of cardiovascular events was significantly different with different numbers of coexisting clinical risk factors.

tients with prior CAD, identified that SDS and cardiac function assessed by Quantitative Gated SPECT were independent and powerful predictors of future cardiac events. ${ }^{42}$ Although cardiac function was assessed by LVEF on echocardiography, the present study also showed the importance of cardiac function and stress MPI (particularly SDS) for predicting cardiovascular events. Cardiovascular events were more frequent in the present study than in the previous study ( $28 \%$ vs. $7.6 \%)$. The present study included a larger number of patients with CKD than the previous study (36\% vs. $1.7 \%$ ), which might explain why cardiovascular events were more frequent in this study.

Aggressive strategies (careful observation, strengthening of sub-optimal therapies and coronary revascularization when myocardial ischemia is observed) are needed as early as possible for patients with prior CAD, particularly for those with coexisting PAD, CKD, current smoking and cardiac dysfunction, regardless of the presence or absence of typical angina symptoms, to prevent adverse cardiac events.

\section{Study Limitations}

An important limitation of this study is that it was conducted as a single-center retrospective trial. Baseline patient characteristics such as clinical data related to symptoms and/or history might not be completely accurate. We could not exclude the possibility that late coronary revascularization might have the physicians' decision-making without consideration of stress MPI results, and the amount of SDS definitely affects the events of late revascularization. Repeated stress MPI was performed in 128 of 354 patients (36\%). Among 24 patients of late revascularization, a second stress MPI was performed in 4 patients to define late revascularization (achieving revascularization within 2 months after second stress MPI). Hence, the first stress MPI might reduce its statistical power. In this study, previous MI was not a predictor of cardiovascular events. The relatively mild myocardial damage (mean $\mathrm{SRS}=5.9 \pm 5.8$ ), preserved LV systolic function (mean LVEF $=57 \pm 13 \%$ ), and remote phase of MI (mean duration from onset of MI to stress MPI: 8.4 \pm 6.4 years and at least 1 year) among our participants might explain why previous MI was not a significant predictor of cardiovascular events. Furthermore, 59\% patients (210/354) had a SSS of $<4$ and $69 \%(245 / 354)$ had SRS of $<4$, both of which are usually regarded as non-significant defects. The severity of a perfusion defect was mild, particularly in patients with previous MI. This might explain why SSS and SRS were not significant predictors of cardiovascular events. If patients with large defects were included, SSS and/or SRS would be independent predictors. Moreover, the difference in SDS was small between patients with and without cardiovascular events (1.4 vs. 0.4). This might decrease the predictive power of SDS. Electrocardiogram-gated SPECT was performed only in 73 patients, in whom 23 cardiovascular events were observed. During the period when stress MPI was assessed (2008-2010), the technique was not performed regularly at our institution due to limited information processing ability of devices. Moreover, we had considered that decreased myocardial thallium washout was suitable for detecting myocardial ischemia, particularly in patients with multiple vessel disease and balanced ischemia. Compared with technetium, thallium was not suitable for QGS. We could not assess the predictive value of cardiac function measured by QGS, including the LV end-diastolic and -systolic volumes, LVEF and peak filling rate because we did not 
use QGS in all patients. In addition, we could not assess smoking status after stress MPI. Data regarding the levels of hemoglobin, albumin, and LDL and HDL cholesterol were available for only $73 \%$ (258/354) patients. These parameters were therefore excluded from multivariate Cox regression analysis. The periods between stress MPI and gathering of laboratory data (ie, examination of ABI or echocardiography) varied, but all these data were obtained within a 3-month period before and after stress MPI. We cannot exclude the possibility that cardiovascular events were missed because of insufficient follow up.

\section{Conclusions}

In conclusion, although the present study has several limitations, we believe our findings provide valuable insights into the usefulness of both background risk factors and grade of myocardial ischemia assessed by stress MPI as predictors of cardiovascular risk in patients with prior CAD. In patients with prior $\mathrm{CAD}$, baseline clinical factors (such as PAD, current smoking, and $\mathrm{CKD}$ ) and cardiac functions might be powerful predictors of cardiovascular events. Among the stress MPI parameters, only the grade of ischemia (SDS) was a predictor of cardiovascular events in patients with prior CAD but without large perfusion defects. In comparison with baseline clinical factors and cardiac function, the grade of the defect assessed by stress MPI might have a relatively limited role in the assessment of cardiovascular prognosis in patients with prior CAD.

\section{Acknowledgments}

We thank Chihiro Hamazaki and Takeharu Ando for their technical assistance in the use of stress MPI.

\section{Disclosures}

Statement of Financial Interest: We have no financial interest/arrangement or affiliation with any organization(s) that could be perceived as a real or apparent conflict of interest in the context of the subject of this study

\section{References}

1. Hachamovitch R, Berman DS, Kiat H, Cohen I, Cabico JA, Friedman $\mathrm{J}$, et al. Exercise myocardial perfusion SPECT in patients without known coronary artery disease: Incremental prognostic value and use in risk stratification. Circulation 1996; 93: 905-914.

2. Hachamovitch R, Berman DS, Shaw LJ, Kiat H, Cohen I, Cabico JA, et al. Incremental prognostic value of myocardial perfusion single photon emission computed tomography for the prediction of cardiac death: Differential stratification for risk of cardiac death and myocardial infarction. Circulation 1998; 97: 535-543.

3. Hachamovitch R, Hayes SW, Friedman JD, Cohen I, Berman DS. Stress myocardial perfusion single-photon emission computed tomography is clinically effective and cost effective in risk stratification of patients with a high likelihood of coronary artery disease (CAD) but no known CAD. J Am Coll Cardiol 2004; 43: 200-208.

4. Sharir T, Germano G, Kavanagh PB, Lai S, Cohen I, Lewin HC, et al. Incremental prognostic value of post-stress left ventricular ejection fraction and volume by gated myocardial perfusion single photon emission computed tomography. Circulation 1999; 100: 10351042 .

5. Nishimura T, Nakajima K, Kusuoka H, Yamashina A, Nishimura S. Prognostic study of risk stratification among Japanese patients with ischemic heart disease using gated myocardial perfusion SPECT: JACCESS study. Eur J Nucl Med Mol Imaging 2008; 35: 319-328.

6. Berman D, Hachamovitch R, Lewin H, Friedman J, Shaw L, Germano G. Risk stratification in coronary artery disease: Implications for stabilization and prevention. Am J Cardiol 1997; 79: 10-16.

7. Berman DS, Kiat H, Friedman JD, Wang FP, van Train K, Matzer L, et al. Separate acquisition rest thallium-201/stress technetium-99m sestamibi dual-isotope myocardial perfusion single-photon emission computed tomography: A clinical validation study. J Am Coll Cardiol 1993; 22: 1455-1464.

8. Berman DS, Hachamovitch R, Kiat H, Cohen I, Cabico JA, Wang $\mathrm{FP}$, et al. Incremental value of prognostic testing in patients with known or suspected ischemic heart disease: A basis for optimal utilization of exercise technetium-99m sestamibi myocardial perfusion single-photon emission computed tomography. J Am Coll Cardiol 1995; 26: 639-647.

9. Galassi AR, Azzarelli S, Tomaselli A, Giosofatto R, Ragusa A, Musumeci S, et al. Incremental prognostic value of technetium- 99 $\mathrm{m}$-tetrofosmin exercise myocardial perfusion imaging for predicting outcomes in patients with suspected or known coronary artery disease. Am J Cardiol 2001; 88: 101 - 116.

10. Ueyama T, Takehana K, Maeba H, Iwasaka T. Prognostic value of normal stress-only technetium-99m myocardial perfusion imaging protocol: Comparison with standard stress-rest protocol. Circ J 2012; 76: $2386-2391$.

11. Nakajima K, Matsuo S, Okuyama C, Hatta T, Tsukamoto K, Nishimura S, et al. Cardiac event risk in Japanese subjects estimated using gated myocardial perfusion imaging, in conjunction with diabetes mellitus and chronic kidney disease. Circ J 2012; 76: 168-175.

12. Nakajima K, Nishimura T. Cardiovascular events in Japan. Lessons from the J-ACCESS multicenter prognostic study using myocardial perfusion imaging. Circ J 2012; 76: 1313-1321.

13. Nanasato M, Morita S, Yoshida R, Niimi T, Sugimoto M, Tsukamoto $\mathrm{K}$, et al. Detection of coronary artery disease using automated quantitation of myocardial perfusion on single-photon emission computed tomography images from patients with angina pectoris without prior myocardial infarction. Circ J 2012; 76: 2280-2282.

14. Shaw LJ, Hendel R, Borges-Neto S, Lauer MS, Alazraki N, Burnette $\mathrm{J}$, et al. Prognostic value of normal exercise and adenosine $99 \mathrm{mTc}-$ tetrofosmin SPECT imaging: Results from the multicenter registry of 4,728 patients. J Nucl Med 2003; 44: 134-139.

15. Elhendy A, Schinkel AF, van Domburg RT, Bax JJ, Valkema R, Poldermans D. Prognostic value of stress Tc-99 $\mathrm{m}$ tetrofosmin SPECT in patients with previous myocardial infarction: impact of scintigraphic extent of coronary artery disease. J Nucl Cardiol 2004; 11: 704709 .

16. Spinelli L, Petretta M, Acampa W, He W, Petretta A, Bonaduce D, et al. Prognostic value of combined assessment of regional left ventricular function and myocardial perfusion by dobutamine and rest gated SPECT in patients with uncomplicated acute myocardial infarction. J Nucl Med 2003; 44: 1023-1029.

17. Acampa W, Spinelli L, Petretta M, De Lauro F, Ibello F, Cuocolo A Prognostic value of myocardial ischemia in patients with uncomplicated acute myocardial infarction: Direct comparison of stress echocardiography and myocardial perfusion imaging. J Nucl Med 2005; 46: 417-423.

18. National Kidney Foundation. K/DOQI clinical practice guidelines for chronic kidney disease: Evaluation, classification, and stratification. Am J Kidney Dis 2002; 39: S1-S266.

19. Newman AB, Shemanski L, Manolio TA, Cushman M, Mittelmark $\mathrm{M}$, Polak JF, et al. Ankle-arm index as a predictor of cardiovascular disease and mortality in the Cardiovascular Health Study: The Cardiovascular Health Study Group. Arterioscler Thromb Vasc Biol 1999; 19: 538-545.

20. Resnick HE, Lindsay RS, McDermott MM, Devereux RB, Jones KL, Fabsitz RR, et al. Relationship of high and low ankle brachial index to all-cause and cardiovascular disease mortality: The Strong Heart Study. Circulation 2004; 109: 733-739.

21. Lee DS, Yeo JS, Chung JK, Lee MM, Lee MC. Transient prolonged stunning induced by dipyridamole and shown on 1- and 24-hour poststress 99 mTc-MIBI gated SPECT. J Nucl Med 2000; 41: 27-35.

22. Hachamovitch R. Assessing the prognostic value of cardiovascular imaging: A statistical exercise or a guide to clinical value and application? Circulation 2009; 120: 1342-1344.

23. Acampa W, Evangelista L, Petretta M, Liuzzi R, Cuocolo A. Usefulness of stress cardiac single-photon emission computed tomographic imaging late after percutaneous coronary intervention for assessing cardiac events and time to such events. Am J Cardiol 2007; 100: 436-441.

24. Furuhashi T, Moroi M, Joki N, Hase H, Masai H, Kunimasa T, et al. Impact of chronic kidney disease and stress myocardial perfusion imaging as a predictor of cardiovascular events. Ann Nucl Med 2011; 25: $616-624$.

25. Aboyans V, Lacroix P, Postil A, Guilloux J, Rolie F, Comu E, et al. Subclinical peripheral arterial disease and incompressible ankle arteries are both long-term prognostic factors in patients undergoing coronary artery bypass grafting. J Am Coll Cardiol 2005; 46: 815820.

26. Van Spall HG, Chong A, Tu JV. Inpatient smoking-cessation counseling and all-cause mortality in patients with acute myocardial infarction. Am Heart J 2007; 154: 213-220.

27. Critchley JA, Capewell S. Mortality risk reduction associated with 
smoking cessation in patients with coronary heart disease: A systematic review. JAMA 2003; 290: 86-97.

28. Mohiuddin SM, Mooss AN, Hunter CB, Grollmes TL, Cloutier DA, Hilleman DE. Intensive smoking cessation intervention reduces mortality in high-risk smokers with cardiovascular disease. Chest 2007 ; 131: $446-452$.

29. Meisinger C1, Döring A, Löwel H; KORA Study Group. Chronic kidney disease and risk of incident myocardial infarction and all-cause and cardiovascular disease mortality in middle-aged men and women from the general population. Eur Heart J 2006; 27: 1245-1250.

30. Go AS, Chertow GM, Fan D, McCulloch CE, Hsu CY. Chronic kidney disease and the risks of death, cardiovascular events, and hospitalization. N Engl J Med 2004; 351: 1296-1305.

31. Manjunath G, Tighiouart H, Ibrahim H, MacLeod B, Salem DN, Griffith JL, et al. Level of kidney function as a risk factor for atherosclerotic cardiovascular outcomes in the community. J Am Coll Cardiol 2003; 41: 47-55.

32. Weiner DE, Tighiouart H, Amin MG, Stark PC, MacLeod B, Griffith $\mathrm{JL}$, et al. Chronic kidney disease as a risk factor for cardiovascular disease and all-cause mortality: A pooled analysis of communitybased studies. J Am Soc Nephrol 2004; 15: 1307-1315.

33. Cohn PF, Gorlin R, Cohn LH, Collins JJ Jr. Left ventricular ejection fraction as a prognostic guide in surgical treatment of coronary and valvular heart disease. Am J Cardiol 1974; 34: 136-141.

34. Battler A, Slutsky R, Karliner J, Froelicher V, Ashburn W, Ross J Jr. Left ventricular ejection fraction and first third ejection fraction early after acute myocardial infarction: Value for predicting mortality and morbidity. Am J Cardiol 1980; 45: 197-202.

35. Curtis JP, Sokol SI, Wang Y, Rathore SS, Ko DT, Jadbabaie F, et al. The association of left ventricular ejection fraction, mortality, and cause of death in stable outpatients with heart failure. $J$ Am Coll
Cardiol 2003; 42: 736-742.

36. Bosch X. Theroux P. Left ventricular ejection fraction to predict early mortality in patients with non-ST segment elevation acute coronary syndromes. Am Heart J 2005; 150: 215-220.

37. Gimelli A, Landi P, Marraccini P, Sicari R, Frumento P, L'Abbate A, et al. Left ventricular ejection fraction measurements: Accuracy and prognostic implications in a large population of patients with known or suspected ischemic heart disease. Int J Cardiovasc Imaging 2008; 24: 793-801.

38. Furuhashi T, Moroi M, Masai H, Kunimasa T, Nakazato R, Fukuda $\mathrm{H}$, et al. Correlation of chronic kidney disease, diabetes and peripheral artery disease with cardiovascular events in patients using stress myocardial perfusion imaging. Ann Nucl Med 2011; 25: 634-642.

39. Sharir T, Germano G, Kang X, Lewin HC, Miranda R, Cohen I, et al. Prediction of myocardial infarction versus cardiac death by gated myocardial perfusion SPECT: Risk stratification by the amount of stress-induced ischemia and the poststress ejection fraction. $J$ Nucl Med 2001; 42: 831-837.

40. Matsumoto N, Sato Y, Suzuki Y, Kasama S, Nakano Y, Kato M, et al. Incremental prognostic value of cardiac function assessed by ECGgated myocardial perfusion SPECT for the prediction of future acute coronary syndrome. Circ J 2008; 72: 2035-2039.

41. Momose M, Nakajima K, Nishimura T. Prognostic significance of stress myocardial gated SPECT among Japanese patients referred for coronary angiography: A study of data from the J-ACCESS database. Eur J Nucl Med Mol Imaging 2009; 36: 1329-1337.

42. Hashimoto A, Nakata T, Wakabayashi T, Kusuoka H, Nishimura T. Incremental prognostic value of stress/rest gated perfusion SPECT in patients with coronary artery disease: Subanalysis of the J-ACCESS study. Circ J 2009; 73: 2288-2293. 